\title{
BMJ Open Protocol for a national cohort study to explore the long-term clinical and patient-reported outcomes and cost- effectiveness of implant-based and autologous breast reconstruction after mastectomy for breast cancer: the brighter study
}

\author{
Leigh Johnson, ${ }^{1}$ Chris Holcombe, ${ }^{2}$ Joe M O'Donoghue, ${ }^{3}$ Ranjeet Jeevan, ${ }^{4}$ \\ John Browne, ${ }^{5}$ Patricia Fairbrother, ${ }^{6}$ Mairead MacKenzie, ${ }^{6}$ Carmel Gulliver-Clarke, ${ }^{7}$ \\ Paul White, ${ }^{8}$ Syed Mohiuddin (D) , ${ }^{9}$ William Hollingworth, ${ }^{9}$ Shelley Potter (D) ${ }^{1,10}$
}

To cite: Johnson $L$, Holcombe C, O'Donoghue JM, et al. Protocol for a national cohort study to explore the long-term clinical and patient-reported outcomes and cost-effectiveness of implantbased and autologous breast reconstruction after mastectomy for breast cancer: the brighter study. BMJ Open 2021;11:e054055. doi:10.1136/ bmjopen-2021-054055

- Prepublication history for this paper is available online. To view these files, please visit the journal online (http://dx.doi. org/10.1136/bmjopen-2021 054055).

Received 07 June 2021 Accepted 29 July 2021

Check for updates

(C) Author(s) (or their employer(s)) 2021. Re-use permitted under CC BY-NC. No commercial re-use. See rights and permissions. Published by BMJ.

For numbered affiliations see end of article.

Correspondence to

Shelley Potter;

shelley.potter@bristol.ac.uk

\section{ABSTRACT}

Introduction Breast reconstruction (BR) is offered to improve quality of life for women with breast cancer undergoing mastectomy. As most women will be long-term breast cancer survivors, high-quality information regarding the long-term outcomes of different BR procedures is essential to support informed decision-making. As different techniques vary considerably in cost, policymakers also require high-quality cost-effectiveness evidence to inform care. The Brighter study aims to explore the long-term clinical and patient-reported outcomes (PROs) of implantbased and autologous BR and use health economic modelling to compare the long-term cost-effectiveness of different reconstructive techniques.

Methods and analysis Women undergoing mastectomy and/or BR following a diagnosis of breast cancer between 1 January 2008 and 31 March 2009 will be identified from hospital episode statistics (HES). Surviving women will be contacted and invited to complete validated PRO measures including the BREAST-Q, EQ-5D-5L and ICECAP-A, or opt out of having their data included in the HES analysis. Longterm clinical outcomes will be explored using HES data. The primary outcome will be rates of revisional surgery between implant-based and autologous procedures. Secondary outcomes will include rates of secondary reconstruction and reconstruction failure. The long-term PROs of implant-based and autologous reconstruction will be compared using BREAST-Q, EQ-5D-5L and ICECAP-A scores. Multivariable regression will be used to examine the relationship between long-term outcomes, patient comorbidities, sociodemographic and treatment factors. A Markov model will be developed using HES and PRO data and published literature to compare the relative long-term cost-effectiveness of implant-based and autologous BR. Ethics and dissemination The Brighter study has been approved by the South-West -Central Bristol Research Ethics Committee (20/SW/0020), and the Confidentiality Advisory Group (20/CAG/0021). Results will be published

\section{Strengths and limitations of this study}

This study will use a robust comprehensive national population-based cohort design to generate high-quality long-term clinical and patient-reported outcome data for different approaches to breast reconstruction.

- Analysis of hospital episode statistics will provide a comprehensive description of the additional NHSfunded procedures performed following primary breast reconstruction in England but is dependent on the quality of procedure coding and will not capture privately funded procedures or procedures performed in devolved nations.

- Women in the cohort underwent breast reconstruction approximately 10 years before the study and engagement and patient-reported outcome response rates may be low.

- Reconstructive practice in the UK has changed over the study period, but a comparison of implant/ expander-based and autologous techniques remains valid and will provide much needed long-term outcome data to inform practice.

in peer-reviewed journals and presented at national meetings. We will work with the professional associations, charities and patient groups to disseminate the results.

\section{INTRODUCTION}

Breast cancer survival is increasing. Over $75 \%$ of the $55000^{1}$ women diagnosed with breast cancer each year in the UK will survive at least 10 years following their diagnosis and two-thirds will survive 20 years or more. Despite advances in breast cancer treatment, 
up to $40 \%^{2}$ of women will require a mastectomy as their surgical treatment. The loss of a breast can profoundly affect women's quality of life ${ }^{3}$ and breast reconstruction (BR) is routinely offered to improve outcomes. ${ }^{4}$ As most women will now be long-term cancer survivors, long-term outcome data are increasingly important to help patients and surgeons make informed decisions about their treatment options.

Decision-making for BR is complex. Many procedures can be performed but these can broadly be divided into implant-based and autologous techniques where the patient's own tissues are used to reconstruct the breast. ${ }^{5}$ Autologous reconstruction can be performed either with pedicled flaps (eg, latissimus dorsi or transverse rectus abdominus myocutaneous flap), where the skin and muscle from the donor-site (back or abdomen) is rotated into the breast area or more complex free-flaps which involve the use of microsurgical techniques to reconstruct the breast using tissue from the abdomen, buttock or thigh. These approaches differ significantly in the duration of the procedure; length of hospital stay and postoperative recovery; number and position of scars and complications. Each procedure can be performed at the time of mastectomy (immediate BR) or delayed (delayed $\mathrm{BR}$ ), often until necessary cancer treatments (chemotherapy and/or radiotherapy) have been completed. ${ }^{5}$ Patients and surgeons need to be able to balance the short-term risks and benefits of different approaches to $\mathrm{BR}$ with the long-term outcomes of surgery to make fully informed decisions. As different techniques vary considerably in cost, policymakers also require high-quality costeffectiveness data to inform the evidence-based provision of care.

Long-term outcome data, however are lacking and high-quality comparative studies are rare.

\section{Long-term clinical outcome studies}

Few studies report long-term clinical outcomes of BR. ${ }^{6}$ Most evidence comes from small, single-centre, retrospective case series with limited follow-up. ${ }^{6}$ Few studies directly compare different procedures ${ }^{6}$ and as outcome reporting is inconsistent and heterogenous, results of individual studies cannot be meaningfully compared.

Large multicentre studies from North America have shown significantly higher complication rates in patients undergoing autologous reconstruction compared with those receiving implant-based procedures at 30 days (12.47\% vs $5.38 \%$; OR: 1.47 (95\% CI: 1.15 to 1.89$))^{7}$; 1 year (any complication $46.9 \%$ vs $24.7 \%$; OR: 2.22 (95\% CI: 1.57 to 3.13$)$ ); major complications requiring readmission/reoperation ( $18.0 \%$ vs $9.3 \%$; OR: 1.75 (95\% CI: 1.19 to 2.58$))^{8}$ and 2 years following surgery, ${ }^{9}$ although the rate of reconstruction failure was higher in patients undergoing implant-based procedures at 2 years. ${ }^{9}$ These relatively short-term outcomes however, need to be balanced against the need for additional surgery for long-term complications such as capsular contracture. Data are limited, but a UK population-based study of
13736 women undergoing immediate BR between 2007 and 2013 demonstrated significantly higher revision rates at 3 years post reconstruction in patients undergoing implant procedures $(55.7 \%)$ than those receiving autologous reconstruction (21.2\%). Furthermore, over a quarter $(27.6 \%)$ of patients who initially had implant $\mathrm{BR}$ required a secondary reconstruction (replacement of their implant with another form of BR) over the same period. ${ }^{10}$ The mean follow-up in this cohort was only 3.7 years, but as the median time to secondary reconstruction is between $4^{11}$ and 6 years ${ }^{12}$, this may underestimate the true scale of the problem, providing further impetus for the long-term outcomes of implant-based and autologous BR to be explored.

\section{Long-term patient reported outcome studies}

Long-term patient-reported outcomes (PROs) comparing implant-based and autologous BR are similarly lacking. Several early studies failed to demonstrate any difference in PROs between procedure types but as these used generic PROs which are not sensitive to BR-related concerns, ${ }^{13}$ the results cannot be relied upon. ${ }^{1415}$

More recently, large multicentre studies have compared implant-based and autologous BR using the BREAST- $^{16}$, a fully-validated BR-specific PRO questionnaire. These studies suggest patients undergoing autologous reconstruction are significantly more satisfied with the outcomes of their surgery and report better healthrelated quality of life (HRQL) than those undergoing implant BR at 12 months ${ }^{17}, 18$ months $^{18}$ and 2 years ${ }^{19}$ postsurgery. Longer-term data are lacking but a singlecentre cross-sectional study of 219 patients undergoing implant-based and autologous BR with a mean follow-up of 6.5 years suggests that patients receiving implant BR are significantly less satisfied with the aesthetic outcome of their surgery over time compared with those undergoing autologous procedures. ${ }^{20}$

The Love Research Army (formerly the US Army of Women) study has recruited 1956 patients with BR with the aim of exploring changes in HRQL over time. ${ }^{21}$ The study has yet to report, but the numbers of women undergoing each procedure type are likely to be relatively small and the self-selected, highly educated patient groups are unlikely to reflect the UK population. ${ }^{21}$ There is therefore a need for long-term PROs comparing implant-based and autologous BR in the UK to inform decision-making and provide evidence for effectiveness.

\section{Cost-effectiveness studies}

Studies comparing the costs and/or cost-effectiveness of implant-based and autologous reconstruction have produced conflicting results. ${ }^{22-29}$ This is likely to reflect variations in the methods used to determine costs of the index procedure and subsequent interventions; complication and revision rates observed in the cohort and the duration of follow-up considered. Cost-effectiveness models using decision analysis methods offer a means of comparing the long-term efficiency of different 
reconstructive techniques. ${ }^{30}$ Decision analytical models, however, require assumptions to be made about the costs and the effectiveness of interventions which must be valid if the results are to be meaningful. Many of the existing models have significant methodological limitations including the use of complication rates reported in the literature ${ }^{23253132}$ or included only limited follow-up. ${ }^{33} 34$

A further methodological challenge is the valuation of the outcomes of BR procedures. ${ }^{35} 36$ Health utilities are usually measured with generic instruments (eg, the EQ-5D-5L) which can be used to estimate quality-adjusted life-years (QALYs). Generic measures have been shown to have limited validity in a BR population, ${ }^{14}$ the EQ-5D-5L was not able to differentiate between implant-based and autologous reconstruction cohorts. ${ }^{37}$ Other studies have used the Breast QALY, a measurement derived from the BREAST-Q questionnaire. ${ }^{23} 31$ The BREAST-Q Utility module is in development and will offer a solution but is not yet ready for use. ${ }^{38}{ }^{39}$ A Breast QALY can be interpreted as 1 year of perfect breast HRQL. ${ }^{31}$ Although this allows efficiency to be measured, the value of a Breast QALY to society is unknown, making interpretation of results for policymakers difficult. The recently developed ICECAP-A $^{40}$ assessment of capability may represent a complementary approach as it focuses on five attributes of well-being (ie, attachment, stability, achievement, enjoyment and autonomy) but has yet to be used in a reconstruction population.

Well-designed studies, and ideally, randomised controlled trials (RCTs), are needed to determine the long-term clinical and cost-effectiveness of different approaches to $\mathrm{BR},{ }^{41}$ but RCTs comparing types of $\mathrm{BR}$ have not been feasible due to patient and surgeon preference, ${ }^{42} 43$ and large prospective cohort studies are timeconsuming and expensive. A population-based cohort approach using a combination of routinely collected clinical outcome data and cross-sectional PROs assessment may offer a timely and efficient approach for estimating the long-term outcomes and costs of BR.

\section{METHODS AND ANALYSIS \\ Primary aim}

The primary aim of the Brighter study is to explore the long-term clinical and cost-effectiveness of implant-based and autologous (pedicled and free-flap) BR to help patients, health professionals and commissioners make more informed decisions about reconstructive breast surgery. Secondary aims are to compare outcomes in patients undergoing immediate and delayed BR, and to explore long-term outcomes in patients undergoing mastectomy only.

\section{Study design}

The Brighter study will consist of three parts:

1. A clinical outcomes cohort study using hospital episode statistics (HES).

2. A PROs study.
3. A cost-effectiveness analysis.

For all analyses, it is anticipated that the following approaches to BR will be compared:

1. Expander/implant reconstruction.

2. Pedicled flap reconstruction with implant/expander.

3. Autologous pedicled flaps.

4. Free-flap reconstruction

Secondary exploratory analyses will compare outcomes in patients undergoing immediate and delayed BR and those undergoing mastectomy only.

\section{Patient and public involvement}

The protocol for the Brighter study has been codeveloped in collaboration with patient representatives from Independent Cancer Patients' Voice (ICPV) to provide much needed long-term BR outcome data to inform decision-making. ${ }^{41}$ There has been extensive patient and public involvement (PPI) in the study design to explore the acceptability of using routinely collected data to identify potential study participants without consent and in the development of patient-facing materials to ensure that study documents are clear and sensitive to the time that has passed since the participants' initial breast cancer diagnosis and treatment. Patient involvement has included focus groups; presentation of the study at local support group meetings and discussion on social media platforms following which study design and materials were iteratively refined. Patient representatives, both members of ICPV, sit on the steering group and will comment on the clinical and PRO data as it is generated and on the health economic model structure to ensure that it is relevant and reflects the patient experience. We will work with our patient representatives to develop lay summaries to disseminate the results to patient groups and charities to ensure that the study findings are accessible and can benefit patient decision-making in the future.

\section{UK National Mastectomy and Breast Reconstruction Audit}

The UK National Mastectomy and Breast Reconstruction Audit (NMBRA) collected data on 18216 women undergoing unilateral mastectomy and/or BR following a diagnosis of breast cancer or ductal carcinoma in situ (DCIS) at 150 English National Health Service (NHS) trusts, six non-English trusts and 114 independent hospitals over a 15-month period between 1 January 2008 and 31 March 2009. ${ }^{18}$ The audit included clinical and PROs and provided a comprehensive picture of the provision and outcomes of care for women undergoing mastectomy and BR surgery in the UK.

The NMBRA represents a unique resource and the current study aimed to follow-up the original NMBRA cohort to provide 10-year clinical and PRO data for this group. Changes to data protection legislation in 2018, however, meant that the original dataset was no longer available for data linkage. Patients meeting the eligibility criteria to be included in the 2008/2009 NMBRA will therefore be identified from HES and will form the cohort for the current study. 


\section{Cohort identification and invitation to participate/opt out}

The Brighter cohort will be identified via NHS HES. The cohort will include all women diagnosed with invasive breast cancer or DCIS (International Classification of Diseases version 10 codes C50 and D05, respectively) undergoing a unilateral mastectomy (Office of Population Censuses and Surveys (OPCS) Classification of Interventions and Procedures (version 4) code B27) and/or BR procedure (B29 (excluding B29.5, reconstruction breast revision); B30.1 (insertion of prosthesis); S48.2 (insertion of skin expander into subcutaneous tissue of breast), B38 (reconstruction of breast using flap of skin of buttock) or B39 (reconstruction of breast using abdominal flap) $)^{44}$ between 1 January 2008 and 31 March 2009. Women will be considered to have undergone immediate $\mathrm{BR}$ if the reconstructive procedure was performed on the same side and same day as the index mastectomy. ${ }^{44}$ Women in the delayed reconstruction cohort will have undergone a reconstructive procedure to the same side as their index mastectomy but at a later date. ${ }^{44}$ The requested extract will include all HES records (Admitted Patient Care, Outpatients, Critical Care and Accident and Emergency) for eligible patients from 1 year prior to surgery, to allow calculation of the Charlson comorbidity index ${ }^{45}$ to the present allowing for 10 years of follow-up data on all secondary care contacts and costs.

Up-to-date contact details will be obtained for surviving women in the cohort via NHS Digital and linkage of the HES extract with the Personal Demographics Service. All women will be sent a study pack including an invitation letter, participant information sheet and consent form with a unique study ID. The study pack will include information about the study including how to participate in the PRO study (see below) and how to opt out of having their clinical data used. Participants will be encouraged to participate/opt out online, but paper versions of the consent form and prepaid reply envelopes will be provided. All HES records of participants who opt out will be permanently deleted. If no response (positive or negative) is received 4 weeks after the initial mailing, a further invitation letter including a unique study ID and link to the study website will be sent to non-responders. If no response is received 4 weeks after the second invitation, it will be assumed that women do not wish to participate in the PRO study but that they have no objection to their data being used in the clinical outcomes analysis. Data for women who have died since their diagnosis will be included in the analysis.

\section{HES population-based cohort study \\ Outcomes}

The primary outcome for the HES clinical outcomes study will be the rates of revisional surgery following different types of immediate and delayed primary BR. Secondary outcomes will include the proportion of patients in each group undergoing secondary reconstruction; rates of reconstruction failure; the proportions of patients undergoing symmetrisation procedures and completion of their reconstruction with nipple reconstruction/ areolar tattooing and the overall numbers of procedures performed in each reconstruction group. Outcomes in women undergoing immediate and delayed BR will be compared.

For women undergoing mastectomy only during the initial study period, the proportion of patients subsequently undergoing delayed reconstruction; the types of reconstruction performed and the timing of delayed reconstruction will be explored.

\section{Definitions}

For the purposes of this study, the following definitions will be used:

Revisional surgery will be defined as any additional surgery to the chest wall, reconstructed breast or donor site, with the same laterality as the initial surgery or involving the donor-site area. An iterative list of OPCS procedure codes identifying revision procedures will be developed and refined in collaboration with expert breast and plastic surgeons and the existing literature to ensure that it is complete and comprehensive prior to commencing the analysis.

Secondary reconstruction is a subgroup of revisions and will be defined as the replacement of the index reconstruction with another, usually different, type of reconstruction with or without reconstruction failure (when the index reconstruction is removed and not replaced). Women undergoing a subsequent expander/implant reconstruction following reconstruction failure will be considered to have undergone a secondary reconstruction. Women undergoing exchange of expander/ implant, in which one expander/implant was removed but immediately replaced with another prosthesis, will be considered to have had a revision of their reconstruction rather than a secondary reconstruction.

Reconstruction failure will be defined as the removal of the reconstruction without replacement.

Symmetrisation surgery will be defined as any procedure performed to the contralateral breast to improve symmetry and will include augmentation, mastopexy and breast reconstruction $R$ procedures.

Procedures performed to complete the reconstruction will include nipple reconstruction, nipple share and nipple/ areolar tattooing.

\section{Statistical analysis}

Analysis of the HES records of all those who have not opted out will begin 2 months after the initial mail out.

Simple summary statistics will be calculated to describe the number, type and timing of revision procedures, reconstruction failures and secondary reconstructions in the whole cohort and by procedure and timing. The number, type and timing of delayed reconstructions performed in the mastectomy cohort will be summarised. Categorical data will be summarised by counts and percentages. Continuous data will be summarised by mean, SD and range if the data are not skewed, and 
median, interquartile range (IQR) and range if the data are skewed.

Rates of revision surgery, reconstruction failure and secondary reconstruction will be calculated for each procedure type and timing and unadjusted rates compared using the $\chi^{2}$ test. Kaplan-Meier survival plots will be used to compare rate and timing of revision and reconstruction failure by procedure type and a log-rank test will be performed. Estimates of revision rates will be adjusted for statistical censoring due to death. These analyses will be extended to include covariates using Cox proportional hazards modelling.

Multivariable logistic regression will be used to examine the relationship between revision surgery, reconstructive failure, secondary reconstruction and sociodemographic (age/ethnicity/deprivation) and treatment factors (BR type and timing/postmastectomy radiotherapy), Charlson Comorbidity Index and treatment centre. These factors have been identified from the literature ${ }^{846-48}$ and previous work as impacting the complication rates of $\mathrm{BR}^{49-51}$

Similar methods will be used to explore the association between the uptake of delayed reconstruction and sociodemographic, tumour-related and treatment factors.

\section{PROs study}

The aim of the PRO study is to collect long-term PRO data on a large cohort of women undergoing simple mastectomy, immediate and delayed reconstruction and to compare long-term PROs in women undergoing implant-based and autologous procedures.

Surviving women will be contacted and invited to complete using three validated questionnaires, the BREAST-Q $^{16}$, EQ-5D-5L, ${ }^{52}$ ICECAP-A $^{40}$ and short studyspecific questionnaire including details of their initial surgery, smoking status; body mass index and any revision surgery they have undergone at a median of 12 years after their initial mastectomy and/or BR. Women will be encouraged to provide consent and complete questionnaires on-line via the REDCap ${ }^{53}$ survey tool but paper copies will be sent to women who prefer this option.

\section{Statistical analysis for the PR0 study}

Each instrument will be scored according to the developers' instructions. Simple summary statistics will be calculated to describe the domain (BREAST-Q) and preference-based (EQ-5D-5L/ICECAP-A) scores for the whole cohort and by procedure type and timing. Continuous data will be summarised by mean, SD and range if the data are not skewed, and median, IQR and range if the data are skewed.

Multivariable regression will be used to examine the relationship between PRO scores and sociodemographic $^{54}$ and treatment factors (BR type and timing/ postmastectomy radiotherapy/revision surgery/reconstruction failure), ${ }^{17-19} 50$ and the Charlson Comorbidity Index.

\section{Cost-effectiveness study}

This component of the study will develop a decision model to compare the lifetime cost-effectiveness of various unilateral implant-based and autologous BR options for women undergoing a BR at the time of mastectomy for breast cancer. The following implant-based and autologous BR options will likely be evaluated:

1. Expander/implant reconstruction.

2. Pedicled flap reconstruction with implant/expander.

3. Autologous pedicled flaps.

4. Free-flap reconstruction

The model's likely structure will involve a decision-tree combined with a Markov process potentially consisting of four health states: 'Primary BR'; 'Secondary BR'; 'BR removed'; and 'Dead'. The decision-tree will estimate the initial surgery costs and complications, while the Markov model will track the lifetime costs and health outcomes. Within the decision-tree, all women with breast cancer will undergo their primary BR at the time of mastectomy, where they will either experience no complications, complications or a failed reconstruction (implant/flap failure). Women with a BR will enter the Markov model via the 'Primary BR' state, while women with a failed reconstruction will enter via the 'BR removed' state. Some women will undergo secondary reconstruction and consequently move into the 'Secondary BR' state; women can have more than one secondary reconstruction. Women could also stay in their current health state or die from any health state. A proportion of women within the 'Primary BR' and 'Secondary BR' states will require subsequent revision(s) and/or completion surgery.

The model will be populated with findings from the HES analysis and PRO study and will be supplemented with additional data from the literature. The baseline hazards of BR removal and secondary BR will be obtained from the HES analysis, while the HRs between the different BR options will likely be obtained from the HES analysis and/or a long-term cohort study in the absence of RCTs. Inpatient costs for revision procedures identified from HES will be included. The model will take the perspective of the UK NHS for costs and calculate QALYs as the primary outcome measure based on the EQ-5D-5L data from the PRO study. We will compare the associations between EQ-5D-5L and BREAST-Q scores and initial $\mathrm{BR}$ procedures to assess whether findings are likely to be sensitive to the choice of outcome measure. Costs and QALYs incurred beyond 12 months will be discounted by $3.5 \%$ per annum. ${ }^{55}$

We will use a cycle length of 3 months to track changes in costs and QALYs. Given that a secondary reconstruction can take place many years after the primary one, ${ }^{12}$ we will run our model over a lifetime horizon. The cycle-specific probability of survival will be derived from age-specific and sex-specific Office of National Statistics (ONS) cancer survival statistics for women diagnosed with breast cancer. ${ }^{56}$

The model will be probabilistic to quantify the uncertainty in the choice between different BR options. 
Cost-effectiveness will be summarised using incremental net monetary benefits and cost-effectiveness acceptability curves to demonstrate how the NHS willingness-to-pay threshold for an additional QALY affects the probability that a strategy is considered cost-effective.

\section{Study timelines}

The study commenced in October 2019. NHS Digital Independent Group Advising on the Release of Data (IGARD) approval was obtained in April 2021. Data from NHS Digital are expected to be received by August 2021. Questionnaires will be sent August/September 2021 and the analysis is planned to be completed early 2022.

\section{ETHICS AND DISSEMINATION}

The Brighter study (IRAS 276030) has been approved by the South West-Central Bristol Research Ethics Committee (REC reference 20/SW/0020), and the Confidentiality Advisory Group (20/CAG/0021).

All potential participants will be informed about the study and given 2 months to opt out of having their data used in the study. Written consent (paper or electronic) will be obtained prior to participation in the PRO study.

Results will be published in peer-reviewed journals and presented at national meetings. Lay summaries will be developed in collaboration with our PPI members and disseminated via the study website and social media. We will work with the professional associations (Association of Breast Surgery, British Association of Plastic Reconstructive and Aesthetic Surgeons and the Royal College of Nursing), charities and patient groups to produce up-to-date patient resources that include information on the need for further surgery over time and the long-term PROs of different reconstruction techniques to help women make more informed decisions about surgery. Policy documents will be produced to promote the evidence-based provision of reconstructive services in the UK and shared with NHS England and the Cancer Alliances.

\section{Author affiliations}

${ }^{1}$ Bristol Centre for Surgical Research, Population Health Sciences, Bristol Medical School, Bristol, UK

${ }^{2}$ Linda McCartney Centre, Liverpool University Hospitals NHS Foundation Trust, Liverpool, UK

${ }^{3}$ Department of Plastic Surgery, Royal Victoria Infirmary, Newcastle upon Tyne, UK

${ }^{4}$ Manchester University NHS Foundation Trust, Manchester, UK

${ }^{5}$ School of Public Health, University College Cork, Cork, Ireland

${ }^{6}$ Independent Cancer Patients' Voice, UK

${ }^{7}$ University Hospitals Sussex NHS Foundation Trust, Sussex, UK

${ }^{8}$ Applied Statistics Group, University of the West of England, Bristol, UK

${ }^{9}$ Population Health Sciences, Bristol Medical School, Bristol, UK

${ }^{10}$ Bristol Breast Care Centre, North Bristol NHS Trust, Bristol, UK

\section{Twitter Shelley Potter @drshelleypotter}

Contributors SP conceived the study and secured funding. All authors contributed to the study design. RJ and JB provided methodological expertise. $\mathrm{CH}, \mathrm{JMO} \mathrm{C}^{\mathrm{D}}$ and CG-C provided clinical input. PW provided statistical expertise. WH and SM designed the health economic analysis and provided health economic expertise. MM and
PF provided expert PPI input. LJ and SP wrote the first draft of the manuscript. All authors read, critically revised and approved the manuscript prior to submission.

Funding This work was funded by a National Institute for Health Research (NIHR) Research for Patient Benefit Programme Grant (PB-PG-0817-20020) and supported by the NIHR Biomedical Research Centre at University Hospitals Bristol and Weston NHS Foundation Trust and the University of Bristol. Shelley Potter is an NIHR Clinician Scientist (CS-2016-16-019). The views expressed in this publication are those of the authors and not necessarily those of the NHS, the National Institute for Health Research or the Department of Health and Social Care.

\section{Competing interests None declared.}

Patient and public involvement Patients and/or the public were involved in the design, or conduct, or reporting or dissemination plans of this research. Refer to the Methods section for further details.

Patient consent for publication Not required.

Provenance and peer review Not commissioned; peer reviewed for ethical and funding approval prior to submission.

Open access This is an open access article distributed in accordance with the Creative Commons Attribution Non Commercial (CC BY-NC 4.0) license, which permits others to distribute, remix, adapt, build upon this work non-commercially, and license their derivative works on different terms, provided the original work is properly cited, appropriate credit is given, any changes made indicated, and the use is non-commercial. See: http://creativecommons.org/licenses/by-nc/4.0/.

\section{ORCID iDs}

Syed Mohiuddin http://orcid.org/0000-0003-4585-3392

Shelley Potter http://orcid.org/0000-0002-6977-312X

\section{REFERENCES}

1 Cancer Research UK. Breast cancer statistics, 2019. Available: https://www.cancerresearchuk.org/health-professional/cancerstatistics/statistics-by-cancer-type/breast-cancer

2 Malata CM, Mclntosh SA, Purushotham AD. Immediate breast reconstruction after mastectomy for cancer. Br J Surg 2000;87:1455-72.

3 Harcourt D, Rumsey N. Psychological aspects of breast reconstruction: a review of the literature. J Adv Nurs 2001;35:477-87.

4 National Institute for Heath and Care Excellence (NICE). Early and locally advanced breast cancer: diagnosis and treatment [NG101] 2018.

5 Thiruchelvam PTR, McNeill F, Jallali N, et al. Post-Mastectomy breast reconstruction. BMJ 2013;347:f5903.

6 Potter S, Brigic A, Whiting PF, et al. Reporting clinical outcomes of breast reconstruction: a systematic review. J Natl Cancer Inst 2011;103:31-46.

7 Mioton LM, Smetona JT, Hanwright PJ, et al. Comparing thirty-day outcomes in prosthetic and autologous breast reconstruction: a multivariate analysis of 13,082 patients? J Plast Reconstr Aesthet Surg 2013;66:917-25.

8 Wilkins EG, Hamill JB, Kim HM, et al. Complications in postmastectomy breast reconstruction: one-year outcomes of the mastectomy reconstruction outcomes Consortium (MROC) study. Ann Surg 2018;267:164-70.

9 Bennett KG, Qi J, Kim HM, et al. Comparison of 2-year complication rates among common techniques for postmastectomy breast reconstruction. JAMA Surg 2018;153:901-8.

10 Mennie J, Mohanna P-N, O'Donoghue J, et al. Rates of secondary surgery following immediate post-mastectomy reconstruction in English NHS hospitals: a national cohort study of 13,736 women. European Journal of Surgical Oncology 2017;43:S2-3.

11 Coriddi M, Shenaq D, Kenworthy E, et al. Autologous breast reconstruction after failed implant-based reconstruction: evaluation of surgical and patient-reported outcomes and quality of life. Plast Reconstr Surg 2019;143:373-9.

12 Tadiparthi S, Staley H, Collis N, et al. An analysis of the motivating and risk factors for conversion from implant-based to total autologous breast reconstruction. Plast Reconstr Surg 2013;132:23-33.

13 Potter S. Investigating the feasibility of randomised clinical trials in breast reconstruction. University of Bristol 2011.

14 Potter S, Thomson HJ, Greenwood RJ, et al. Health-Related quality of life assessment after breast reconstruction. Br J Surg 2009;96:613-20. 
15 Winters ZE, Benson JR, Pusic AL. A systematic review of the clinical evidence to guide treatment recommendations in breast reconstruction based on patient- reported outcome measures and health-related quality of life. Ann Surg 2010;252:929-42.

16 Pusic AL, Klassen AF, Scott AM, et al. Development of a new patientreported outcome measure for breast surgery: the BREAST-Q. Plast Reconstr Surg 2009;124:345-53.

17 Pusic AL, Matros E, Fine N, et al. Patient-reported outcomes 1 year after immediate breast reconstruction: results of the mastectomy reconstruction outcomes Consortium study. J Clin Oncol 2017;35:2499-506.

18 Jeevan R, Cromwell DA, Browne JP, et al. Findings of a national comparative audit of mastectomy and breast reconstruction surgery in England. J Plast Reconstr Aesthet Surg 2014;67:1333-44.

19 Santosa KB, Qi J, Kim HM, et al. Long-Term patient-reported outcomes in postmastectomy breast reconstruction. JAMA Surg 2018:153:891-9.

20 Hu ES, Pusic AL, Waljee JF, et al. Patient-reported aesthetic satisfaction with breast reconstruction during the long-term survivorship period. Plast Reconstr Surg 2009;124:1-8.

21 Fuzesi S, Cano SJ, Klassen AF, et al. Validation of the electronic version of the BREAST-Q in the army of women study. Breast 2017;33:44-9.

22 Atherton DD, Hills AJ, Moradi P, et al. The economic viability of breast reconstruction in the UK: comparison of a single surgeon's experience of implant; LD; TRAM and DIEP based reconstructions in 274 patients. J Plast Reconstr Aesthet Surg 2011;64:710-5.

23 Matros E, Albornoz CR, Razdan SN, et al. Cost-effectiveness analysis of implants versus autologous perforator flaps using the BREAST-Q. Plast Reconstr Surg 2015;135:937-46.

24 Damen THC, Wei W, Mureau MAM, et al. Medium-term cost analysis of breast reconstructions in a single Dutch centre: a comparison of implants, implants preceded by tissue expansion, LD transpositions and DIEP flaps. J Plast Reconstr Aesthet Surg 2011;64:1043-53

25 Grover R, Padula WV, Van Vliet M, et al. Comparing five alternative methods of breast reconstruction surgery: a cost-effectiveness analysis. Plast Reconstr Surg 2013;132:709e-23.

26 Fischer JP, Wes AM, Nelson JA, et al. Propensity-matched, longitudinal outcomes analysis of complications and cost: comparing abdominal free flaps and implant-based breast reconstruction. J Am Coll Surg 2014;219:303-12.

27 Lagares-Borrego A, Gacto-Sanchez P, Infante-Cossio P, et al. A comparison of long-term cost and clinical outcomes between the two-stage sequence expander/prosthesis and autologous deep inferior epigastric flap methods for breast reconstruction in a public hospital. J Plast Reconstr Aesthet Surg 2016;69:196-205.

28 Lemaine V, Schilz SR, Van Houten HK, et al. Autologous breast reconstruction versus implant-based reconstruction: how do longterm costs and health care use compare? Plast Reconstr Surg 2020;145:303-11.

29 Kouwenberg CAE, Mureau MAM, Kranenburg LW, et al. Cost-Utility analysis of four common surgical treatment pathways for breast cancer. Eur J Surg Oncol 2021;47:1299-1308.

30 Caro JJ, Briggs AH, Siebert U, et al. Modeling good research practices-overview: a report of the ISPOR-SMDM Modeling Good Research Practices Task Force--1. Value Health 2012;15:796-803.

31 Razdan SN, Cordeiro PG, Albornoz CR, et al. Cost-effectiveness analysis of breast reconstruction options in the setting of postmastectomy radiotherapy using the BREAST-Q. Plast Reconstr Surg 2016;137:510e-7.

32 Tran BNN, Fadayomi A, Lin SJ, et al. Cost analysis of postmastectomy reconstruction: a comparison of two staged implant reconstruction using tissue expander and acellular dermal matrix with abdominal-based perforator free flaps. J Surg Oncol 2017:116:439-47.

33 Fischer JP, Fox JP, Nelson JA, et al. A longitudinal assessment of outcomes and healthcare resource utilization after immediate breast reconstruction-comparing Implant- and Autologous-based breast reconstruction. Ann Surg 2015;262:692-9.

34 Aliu O, Zhong L, Chetta MD, et al. Comparing health care resource use between implant and autologous reconstruction of the irradiated breast: a national Claims-Based assessment. Plast Reconstr Surg 2017:139:1224e-31.
35 Cano S, Klassen AF, Scott A, et al. Health outcome and economic measurement in breast cancer surgery: challenges and opportunities. Expert Rev Pharmacoecon Outcomes Res 2010;10:583-94.

36 Preminger BA, Pusic AL, McCarthy CM, et al. How should quality-of-life data be incorporated into a cost analysis of breast reconstruction? A consideration of implant versus free TRAM flap procedures. Plast Reconstr Surg 2008;121:1075-82.

37 Kouwenberg CAE, Kranenburg LW, Visser MS, et al. "The validity of the EQ-5D-5L in measuring quality of life benefits of breast reconstruction". J Plast Reconstr Aesthet Surg 2019;72:52-61.

38 Kaur M, Pusic AL, Cano SJ, et al. International phase 1 study protocol to develop a health state classification system for a preference-based measure for women with breast cancer: the BREAST-Q utility module. BMJ Open 2020;10:e034451.

39 Kaur MN, Klassen AF, Xie F, et al. An international mixed methods study to develop a new preference-based measure for women with breast cancer: the BREAST-Q utility module. BMC Womens Health 2021;21:8.

40 Al-Janabi H, Flynn TN, Coast J. Development of a self-report measure of capability wellbeing for adults: the ICECAP-A. Qual Life Res 2012;21:167-76.

41 Cutress RI, Mclntosh SA, Potter S, et al. Opportunities and priorities for breast surgical research. Lancet Oncol 2018;19:e521-33.

42 Potter S, Mills N, Cawthorn SJ, et al. Time to be brave: is educating surgeons the key to unlocking the potential of randomised clinical trials in surgery? A qualitative study. Trials 2014;15:80.

43 Winters ZE, Emson M, Griffin C, et al. Learning from the quest multicentre feasibility randomization trials in breast reconstruction after mastectomy. Br J Surg 2015;102:45-56.

44 Mennie JC, Mohanna P-N, O'Donoghue JM, et al. National trends in immediate and delayed post-mastectomy reconstruction procedures in England: a seven-year population-based cohort study. Eur J Surg Oncol 2017;43:52-61.

45 Armitage JN, van der Meulen JH, Royal College of Surgeons Comorbidity Consensus Group. Identifying co-morbidity in surgical patients using administrative data with the Royal College of surgeons Charlson score. Br J Surg 2010;97:772-81.

46 Berlin NL, Tandon VJ, Qi J, et al. Hospital variations in clinical complications and patient-reported outcomes at 2 years after immediate breast reconstruction. Ann Surg 2019;269:959-65

47 Berlin NL, Momoh AO, Qi J, et al. Racial and ethnic variations in one-year clinical and patient-reported outcomes following breast reconstruction. Am J Surg 2017;214:312-7.

48 Jagsi R, Momoh AO, Qi J, et al. Impact of radiotherapy on complications and patient-reported outcomes after breast reconstruction. J Natl Cancer Inst 2018;110:157-65.

49 Browne JP, Jeevan R, Gulliver-Clarke C, et al. The association between complications and quality of life after mastectomy and breast reconstruction for breast cancer. Cancer 2017;123:3460-7.

50 Jeevan R, Browne JP, Gulliver-Clarke C, et al. Surgical determinants of patient-reported outcomes following postmastectomy reconstruction in women with breast cancer. Plast Reconstr Surg 2017:139:1036e-45.

51 Jeevan R, Browne JP, Pereira J, et al. Socioeconomic deprivation and inpatient complication rates following mastectomy and breast reconstruction surgery. Br J Surg 2015;102:1064-70.

52 Herdman M, Gudex C, Lloyd A, et al. Development and preliminary testing of the new five-level version of EQ-5D (EQ-5D-5L). Qual Life Res 2011;20:1727-36.

53 Harris PA, Taylor R, Thielke R, et al. Research electronic data capture (REDCap)-a metadata-driven methodology and workflow process for providing translational research informatics support. J Biomed Inform 2009;42:377-81.

54 Santosa KB, Qi J, Kim HM, et al. Effect of patient age on outcomes in breast reconstruction: results from a multicenter prospective study. J Am Coll Surg 2016;223:745-54.

55 National Institute for Health and Care Excellence (NICE). Guide to the methods of technology appraisal, 2013. Available: https://www. nice.org.uk/process/pmg9/resources/guide-to-the-methods-oftechnology-appraisal-2013-pdf-2007975843781

56 Office for National Statistics. Cancer survival in England: adults diagnosed between 2013 and 2017 and followed up to 2018, 2019. Available: https://www.ons.gov.uk/peoplepopulationandcommunity/ healthandsocialcare/conditionsanddiseases/datasets/cancersurviv alratescancersurvivalinenglandadultsdiagnosed 